\title{
Coupling a Lattice Boltzmann and a Finite Difference Scheme
}

\author{
Paul Albuquerque ${ }^{1,2}$, Davide Alemani ${ }^{3}$, Bastien Chopard ${ }^{1}$, and Pierre Leone ${ }^{1,2}$ \\ 1 Computer Science Department, University of Geneva, 1211 Geneva 4, Switzerland \\ 2 LII, Ecole d'Ingénieurs de Genève, HES-SO, 1202 Geneva, Switzerland \\ ${ }^{3}$ CABE, University of Geneva, 1211 Geneva 4, Switzerland
}

\begin{abstract}
We show how a lattice Boltzmann (LB) scheme can be spatially coupled with a finite difference (FD) scheme in order to solve the same problem. The typical situation we consider is a computational domain which is partitioned in two regions. The same spatio-temporal physical process extends over the full domain but a different numerical method is used over each region. At the interface of the subdomains, the LB and FD must be connected so as to ensure a perfect continuity of the physical quantities. We derive the theoretical concepts, which allow us to link both methods in the case of a diffusion process, and validate them with numerical simulations on a $2 \mathrm{D}$ domain.
\end{abstract}

\section{Introduction}

Many physical systems include phenomena at different time and space scales. Their description in terms of a numerical model is therefore a difficult task as often the same numerical scheme is not efficient over the full range of scales. In addition, it occurs that various physical processes take place and couple different parts of the system. Again, it is challenging to devise a numerical method which is able to efficiently deal with such constraints.

Here we consider the case of a spatially extended system in which separate spatial regions are treated with different numerical schemes. The motivation is that, depending on the nature of each region, optimal efficiency is obtained with different numerical methods.

For instance the lattice Boltzmann (LB) method [1] has a more detailed microscopic description than a finite difference (FD) scheme because the LB approach includes the molecular velocity of the particles. In addition, important physical quantities, such as the stress tensor, or particle current, are directly obtained from the local information. However, the LB scheme may require more memory than the corresponding FD scheme. Another motivation is that boundary conditions are more or less naturally imposed on a given numerical scheme. Therefore, in order to improve the global quality of the numerical solution, it may be quite efficient to assume that several solvers are coupled.

Obviously, this coupling should not produce any discontinuities at the interface between regions that are treated with the LB or the FD method. Since each 
scheme uses different variables, it is crucial to understand how the LB set of variables is related to the FD set, and conversely.

Our solution follows the same argument as developed in 2] for a multigrid LB scheme: the connection between the full set of LB variables and the standard macroscopic physical quantities is based on the splitting of the particle distribution functions in an equilibrium and a nonequilibrium part. The former is built on the physical quantities and the latter on the gradient of these quantities. This observation is quite general and could be used to couple any scheme (e.g. finite volume or finite element) with any LB method. Indeed, if the two schemes use different variables, one must compute the particle distribution functions starting from physical quantities known from another solver or from an analytical expression. Here we will only consider the case of a diffusion process on a given domain which is solved by the LB approach on a chosen subdomain and with a FD solver on the rest of the domain.

Hybrid methods have already been proposed in the literature. In 3 a finite volume and a finite element method are combined and then coupled with a finite difference time domain solver for the wave equation, allowing the simulation of wave propagation in complex 3D geometry. In 4], the LB method is coupled with a molecular dynamics simulation of polymers. However, to our knowledge, the FD and LB schemes have never been coupled across adjacent regions.

The paper is organized as follows. In section 2 we briefly introduce the LB approach (we assume that the reader is familiar with the FD method). The special case of a diffusion process is discussed in section 3 In particular, it is shown how the Chapman-Enskog expansion offers a mapping between the LB variables and the macroscopic quantities and their spatial derivatives. In section 4 we describe the coupling algorithm. Then, in section 5 we perform a numerical simulation to demonstrate that the proposed coupling is correct. Finally, section 6]summarizes our findings and draws some conclusions.

\section{The Lattice Boltzmann Approach}

A lattice Boltzmann (LB) model [1,5] describes a physical system in terms of a mesoscopic dynamics: fictitious particles move on a regular lattice, synchronously at discrete time steps. An interaction is defined between the particles that meet simultaneously at the same lattice site. Particles obey collision rules which reproduce, in the macroscopic limit, an equation of physics.

A LB model can be interpreted as a discretization of the Boltzmann transport equation on a regular lattice of spacing $\Delta r$ and with discrete time step $\Delta t$. The possible velocities for the pseudo-particles are the vectors $\boldsymbol{v}_{i}$. They are chosen so as to match the lattice direction: if $\boldsymbol{r}$ is a lattice site, $\boldsymbol{r}+\boldsymbol{v}_{i} \Delta t$ is also a lattice point. We thus consider a dynamics with $z+1$ possible velocities, where $z$ is the lattice coordination number and $\boldsymbol{v}_{0}=0$ describe the population of rest particles.

For isotropy reasons the lattice topology must satisfy the conditions

$$
\sum_{i} v_{i \alpha}=0 \text { and } \sum_{i} v_{i \alpha} v_{i \beta}=v^{2} C_{2} \delta_{\alpha \beta}
$$


where $C_{2}$ is a numerical coefficient which depends on the lattice topology. The greek indices label the spatial dimensions and $v=\Delta t / \Delta r$. The first condition follows from the fact that if $\boldsymbol{v}_{i}$ is a possible velocity, then so is $-\boldsymbol{v}_{i}$.

In the LB approach a physical system is described through density distribution functions $f_{i}(\boldsymbol{r}, t)$. For hydrodynamics and diffusion processes, $f_{i}(\boldsymbol{r}, t)$ represents the distribution of particles entering site $\boldsymbol{r}$ at time $t$ and moving in direction $\boldsymbol{v}_{i}$. For the wave model, the interpretation is less obvious as $f_{i}$ can be positive as well as negative. Physical quantities can be defined from moments of these distributions. For instance, the local density is obtained by $\rho=\sum_{i=0}^{z} f_{i}$.

A LB model can be determined by specifying a lattice, a kinetic equation and an equilibrium distribution. In its simplest form (BGK model), the dynamics can be written as a relaxation to a given local equilibrium

$$
f_{i}\left(\boldsymbol{r}+\boldsymbol{v}_{i} \Delta t, t+\Delta t\right)-f_{i}(\boldsymbol{r}, t)=\frac{1}{\tau}\left(f_{i}^{e q}(\boldsymbol{r}, t)-f_{i}(\boldsymbol{r}, t)\right)
$$

where $\tau$ is a relaxation time, which is a free parameter of the model. The local equilibrium solution $f_{i}^{e q}$ contains all the information concerning the physical process investigated. It changes according to whether we consider hydrodynamics, diffusion or wave propagation.

\section{The Lattice Boltzmann Diffusion Model}

Diffusion processes were first modeled using a cellular automata approach [6]. The scheme was then extended to a LB dynamics with a BGK collision term [1] [5,7].

From now on, we assume that the diffusion process takes place on a $d$ dimensionnal square lattice. The key point is to choose correctly the local equilibrium $f_{i}^{e q}$ so that the diffusion equation for the particle density $\rho=\sum_{i=0}^{2 d} f_{i}$ can be derived from eq. (2). Since the particle density $\rho$ is the only conserved quantity in a diffusive process, the local equilibrium $f_{i}^{e q}$ is taken to be $f_{i}^{e q}(\boldsymbol{r}, t)=\rho(\boldsymbol{r}, t) / 2 d$ so that $\rho$ is indeed conserved and $f_{i}^{e q}$ depends on $\boldsymbol{r}$ and $t$ only through the conserved quantities 15].

Let us now assume that $\Delta t$ is small (and constant in all our computations). Taylor expanding the left hand side of eq. (2) up to second order, we get

$$
\begin{gathered}
\Delta t\left(\boldsymbol{v}_{i} \cdot \nabla\right) f_{i}(\boldsymbol{r}, t)+\Delta t \partial_{t} f_{i}(\boldsymbol{r}, t)+\frac{\Delta t^{2}}{2} \partial_{t}^{2} f_{i}(\boldsymbol{r}, t)+ \\
\Delta t^{2}\left(\boldsymbol{v}_{i} \cdot \nabla\right) \partial_{t} f_{i}(\boldsymbol{r}, t)+\frac{\Delta t^{2}}{2}\left(\boldsymbol{v}_{i} \cdot \nabla\right)^{2} f_{i}(\boldsymbol{r}, t)=\frac{1}{\tau}\left(f_{i}^{e q}(\boldsymbol{r}, t)-f_{i}(\boldsymbol{r}, t)\right) .
\end{gathered}
$$

We then use the multiscale Chapman-Enskog expansion to solve eq. (3). Thus, we set $f_{i}=f_{i}^{(0)}+f_{i}^{(1)}+f_{i}^{(2)}+\ldots$ and introduce next a small parameter $\epsilon$ along with the change of coordinates $(\boldsymbol{r}, t) \mapsto\left(\boldsymbol{r}_{1}, t_{1}, t_{2}\right)=\left(\epsilon \boldsymbol{r}, \epsilon t, \epsilon^{2} t\right)$. We also consider a new function $f_{i}^{\epsilon}\left(\boldsymbol{r}_{1}, t_{1}, t_{2}\right)$ which satisfies $f_{i}(\boldsymbol{r}, t)=f_{i}^{\epsilon}\left(\epsilon \boldsymbol{r}, \epsilon t, \epsilon^{2} t\right)$. After formally substituting $f_{i} \longrightarrow f_{i}^{\epsilon}, \nabla_{r} \longrightarrow \epsilon \nabla_{r_{1}}, \partial_{t} \longrightarrow \epsilon \partial_{t_{1}}+\epsilon^{2} \partial_{t_{2}}$, into eq. (3), we obtain a new equation for $f_{i}^{\epsilon}$. 
To find an asymptotic series solution, we expand $f_{i}^{\epsilon}$ in powers of $\epsilon$

$$
f_{i}^{\epsilon}\left(\boldsymbol{r}_{1}, t_{1}, t_{2}\right)=f_{i}^{\epsilon(0)}\left(\boldsymbol{r}_{1}, t_{1}, t_{2}\right)+\epsilon f_{i}^{\epsilon(1)}\left(\boldsymbol{r}_{1}, t_{1}, t_{2}\right)+\epsilon^{2} f_{i}^{\epsilon(2)}\left(\boldsymbol{r}_{1}, t_{1}, t_{2}\right)+\ldots
$$

By introducing (44) into the equation for $f_{i}^{\epsilon}$, we can recursively determine the $f_{i}^{\epsilon(j)}$ s. Using particle density conservation and the first equation in (1), we get at zero and first order

$$
f_{i}^{(0)}(\boldsymbol{r}, t)=\frac{\rho(\boldsymbol{r}, t)}{2 d}=f_{i}^{e q}(\boldsymbol{r}, t), \quad f_{i}^{(1)}=-\frac{\tau \Delta t}{2 d} \boldsymbol{v}_{i} \cdot \nabla \rho(r, t) .
$$

It is still worth noticing, with respect to the macrocospic behaviour of our model, that the particle density $\rho(\boldsymbol{r}, t)$ satisfies the diffusion equation. Indeed, using the second equation in (10), we get

$$
\partial_{t} \rho(\boldsymbol{r}, t)=\frac{C_{2} v^{2} \Delta t}{2 d}\left(\tau-\frac{1}{2}\right) \nabla^{2} \rho(\boldsymbol{r}, t),
$$

with diffusion coefficient $D=(\tau-1 / 2) C_{2} v^{2} \Delta t /(2 d)$.

\section{The Coupling Algorithm}

The result of the previous section shows that the LB variables can be written as $f_{i}=f_{i}^{e q}+f_{i}^{n e q}$, where

$$
f_{i}^{e q}=f_{i}^{(0)}=\frac{\rho(\boldsymbol{r}, t)}{2 d}, \quad f_{i}^{n e q} \approx f_{i}^{(1)}=-\frac{\tau \Delta t}{2 d} \boldsymbol{v}_{i} \cdot \nabla \rho(r, t) .
$$

These relations give a dictionary to go from the standard description of diffusion, where only $\rho$ is considered, to the LB description. Note that the inverse connection is straightforward since we always have, by definition, $\rho=\sum_{i} f_{i}$. It must be clear that the LB scheme requires more information on the physical variables because it contains more degrees of freedom.

To make the coupling between a FD and a LB scheme more explicit, we now consider the situation of a two-dimensional diffusion problem $\partial_{t} \rho=D \nabla^{2} \rho$ on a square domain $\Omega \subset \mathbb{R}^{2}$ of size $L_{x} \times L_{y}$. We cut $\Omega$ into two parts $\Omega_{1}$ and $\Omega_{2}$ such that $\Omega=\Omega_{1} \cup \Omega_{2}$. We apply in $\Omega_{1}$ the FD method and in $\Omega_{2}$ the LB method (see fig. 1). We assume here that the same mesh is used for both methods.

We define the quantities $\boldsymbol{v}_{i}, i=1, \ldots, 4$, as vectors pointing in the four lattice directions: right, up, left and down. Their lengths are such that $\boldsymbol{v}_{i} \Delta t$ connects the neighboring sites along direction $i$.

For the points $\boldsymbol{r} \in \Omega_{1}$, we apply the FD scheme to the diffusion equation

$$
\rho(\boldsymbol{r}, t+\Delta t)=\rho(\boldsymbol{r}, t)+\frac{\Delta t}{\Delta r^{2}} D \sum_{i=1}^{4}\left(\rho\left(\boldsymbol{r}+\boldsymbol{v}_{i} \Delta t, t\right)-\rho(\boldsymbol{r}, t)\right),
$$

whereas the points $r \in \Omega_{2}$ are treated with the LB scheme given by eq. (2). There are several ways to define the interface between the two regions, $\Omega_{1}$ and 

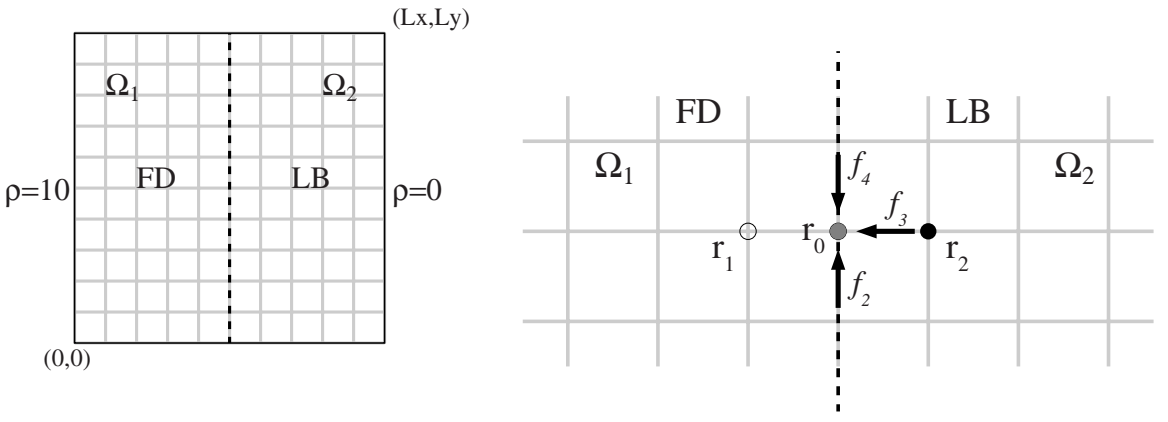

Fig. 1. Left: The computational grid and its partioning into a subdomain $\Omega_{1}$, where the FD scheme is applied, and a subdomain $\Omega_{2}$, where the LB scheme is used. The boundary conditions we impose for the diffusion process are $\rho=10$ on the left wall, $\rho=0$ on the right one and periodic along the vertical axis. Right: The point $\boldsymbol{r}_{1}$ (white circle), resp. $\boldsymbol{r}_{2}$ (black circle), is completely treated with the FD, resp. LB, scheme, and $\boldsymbol{r}_{0}$ (gray circle) is updated with both methods. The figure shows that for $\boldsymbol{r}_{0}$, the distribution $f_{1}$ is unknown (because it should come out of $\boldsymbol{r}_{1}$ ). Thus, we use our coupling algorithm to compute it

$\Omega_{2}$. Here, we assume an overlap so that a point $\boldsymbol{r}_{0}$ at the interface belongs to both $\Omega_{1}$ and $\Omega_{2}$ (see fig. 1 . On such points $\boldsymbol{r}_{0}$, the LB variables as well as the FD variables are computed. We denote by $\boldsymbol{r}_{1} \in \Omega_{1}$ and $\boldsymbol{r}_{2} \in \Omega_{2}$, two neighbors of a site $\boldsymbol{r}_{0}$ of the interface. According to fig. 1 and eq. (5), the calculation of $\rho\left(\boldsymbol{r}_{0}, t+\Delta t\right)$ requires the knowledge of $\rho\left(\boldsymbol{r}_{2}, t\right)$, where $\boldsymbol{r}_{2}$ is only subject to the LB scheme. However, the LB scheme offers naturally this quantity as $\rho\left(\boldsymbol{r}_{2}, t\right)=\sum_{i=1}^{4} f_{i}\left(\boldsymbol{r}_{2}, t\right)$. Therefore, the coupling of a FD site to a LB site is straightforward. The reverse is a bit more involved. In order to update the LB scheme at $\boldsymbol{r}_{0}$ and time $t+\Delta t$, we need $f_{1}\left(\boldsymbol{r}_{0}, t\right)$. This quantity is not known because the site $\boldsymbol{r}_{1}$ is only treated with the FD scheme. Indeed, if the LB scheme had been applied to the full domain, then $f_{1}\left(\boldsymbol{r}_{0}, t\right)$ would have been propagated from lattice site $\boldsymbol{r}_{1}$. However, the value of $f_{1}\left(\boldsymbol{r}_{0}, t\right)$ can be computed from our dictionary $f_{1}\left(\boldsymbol{r}_{0}, t\right)=f_{1}^{(0)}\left(\boldsymbol{r}_{0}, t\right)+f_{1}^{(1)}\left(\boldsymbol{r}_{0}, t\right)$ with $f_{1}^{(0)}\left(\boldsymbol{r}_{0}, t\right)=\rho\left(\boldsymbol{r}_{0}, t\right) / 4$ and $f_{1}^{(1)}\left(\boldsymbol{r}_{0}, t\right)=-(\tau \Delta t / 4) \boldsymbol{v}_{i} \cdot \nabla \rho\left(\boldsymbol{r}_{0}, t\right)$. In order to obtain $\nabla \rho\left(\boldsymbol{r}_{0}, t\right)$, we use a second order finite difference approximation of $\rho$ over the nearest neighbors

$$
\nabla \rho\left(\boldsymbol{r}_{0}, t\right)=\frac{\rho\left(\boldsymbol{r}_{2}, t\right)-\rho\left(\boldsymbol{r}_{1}, t\right)}{2 \Delta r} .
$$

Note that in the particular case where only one $f_{i}$ is missing, the connection can be made in a simpler way. As $\rho=\sum_{i} f_{i}$ is known from the FD calculation, and $f_{2}, f_{3}$ and $f_{4}$ are known from the LB calculation, one has $f_{1}=\rho-f_{2}-f_{3}-f_{4}$. In the simple case described here, this approach gives a correct coupling. However, it no longer works if the interface between $\Omega_{1}$ and $\Omega_{2}$ is irregular because the previous expression is not sufficient to determine more than one $f_{i}$. 


\section{Numerical Validation}

To validate the coupling algorithm proposed in the previous section, we performed the simulation of a source-sink problem on the geometry defined in fig. [ The domain size is $L_{x}=L_{y}=30$ in lattice units. The boundary conditions are $\rho((0, y), t)=10$ and $\rho\left(\left(L_{x}, y\right), t\right)=0$ on the left and right sides of $\Omega$ and the initial conditions are $\rho((0, y), 0)=10$ and $\rho((x, y), 0)=0$ for $x \neq 0$. On the lower and upper walls, we apply periodic conditions $\rho\left(\left(x, L_{y}+1\right), t\right)=\rho((x, 0), t)$ and $\rho((x,-1), t)=\rho\left(\left(x, L_{y}\right), t\right)$. The vertical interface between the subdomains is positioned at $x=6$, with the FD scheme over the left region and the LB over the right one. Hence, the geometry is actually 1-dimensional.

This simple set-up with the above boundary and initial conditions allows for an analytic solution to the diffusion equation for the time-dependent dynamics,

$$
\begin{aligned}
\rho((x, y), t)= & \rho_{0} \sum_{i=0}^{\infty} \operatorname{erfc}\left(\frac{\mathrm{L}_{\mathrm{x}}\left(1-\left(1-\mathrm{x} / \mathrm{L}_{\mathrm{x}}\right)+2 \mathrm{i}\right)}{2 \sqrt{\mathrm{Dt}}}\right) \\
& -\rho_{0} \sum_{i=0}^{\infty} \operatorname{erfc}\left(\frac{\mathrm{L}_{\mathrm{x}}\left(1-\left(1+\mathrm{x} / \mathrm{L}_{\mathrm{x}}\right)+2 \mathrm{i}\right)}{2 \sqrt{\mathrm{Dt}}}\right)
\end{aligned}
$$

where $\operatorname{erfc}(\mathrm{v})=\int_{\mathrm{v}}^{\infty} \mathrm{e}^{-\mathrm{u}^{2}}$ du and $D$ is the diffusion coefficient (here $\left.\rho_{0}=10\right)$.

After several iterations, the system converges to a steady state in which the density profile is expected to be a linear function of $x$ satisfying the boundary conditions. To illustrate the importance of a correct coupling at the subdomain interface, we plot in figs. 2 $\mathrm{a}, 2 \mathrm{~b}, 2 \mathrm{k}$ (left) the density profile obtained from the numerical solution with the full coupling $f_{1}=f_{1}^{(0)}+f_{1}^{(1)}$, the one without the gradient correction, i.e. with the approximation $f_{1} \approx f_{1}^{(0)}$, and the theoretical profile. We also plot in figs. 2], 2 $b$, 2 $\mathrm{c}$ (right) the error with respect to the analytic solution. From the breaking of the slope observed in fig. 2k (left) we conclude that the gradient correction is necessary to obtain the correct density profile and continuity of the particle current.

We computed the relative error in the $L^{2}$-norm for the lattice resolution used in the simulation. For 100 (resp. 500, 5000) time steps, we get a 1.2\% (resp. $0.6 \%$, $0.3 \%$ ) relative error for the full coupling. We expect the level of accuracy of our mapping to be the same as the LB itself: second order in the lattice spacing.

\section{Conclusion}

In this work, a LB scheme is spatially coupled to a FD scheme on a computational domain partitioned in two regions. We propose a way to relate the LB distribution functions $f_{i}$ with the classical physical quantities and their derivatives. This is a first step towards coupling correctly a LB scheme with another method. Hence, to focus on the methodology only, we consider the simplest possible situation: a diffusion process solved by the LB approach on one region and 
(a)
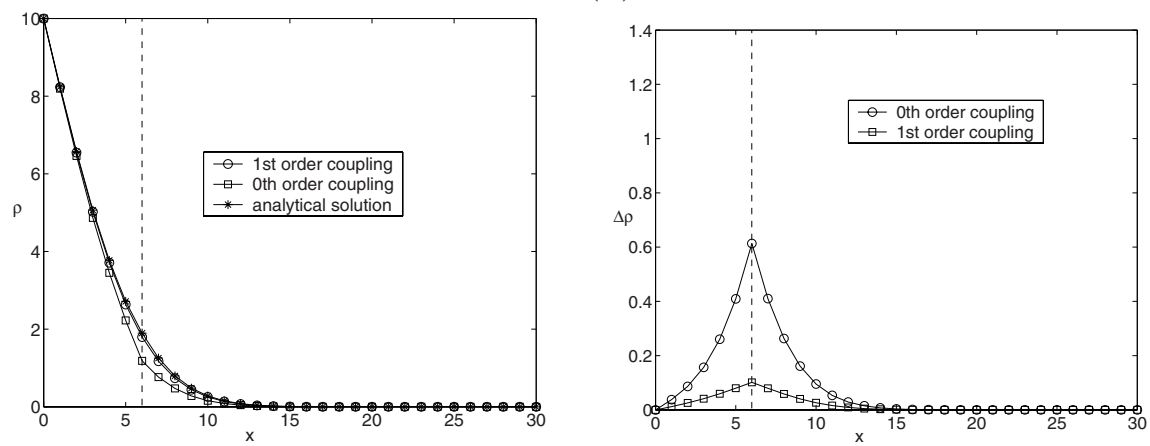

(b)
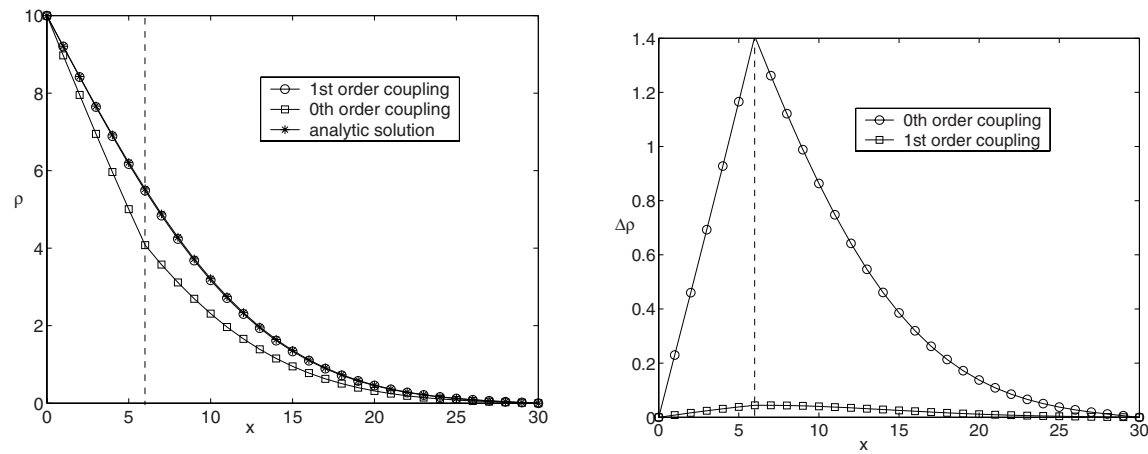

(c)
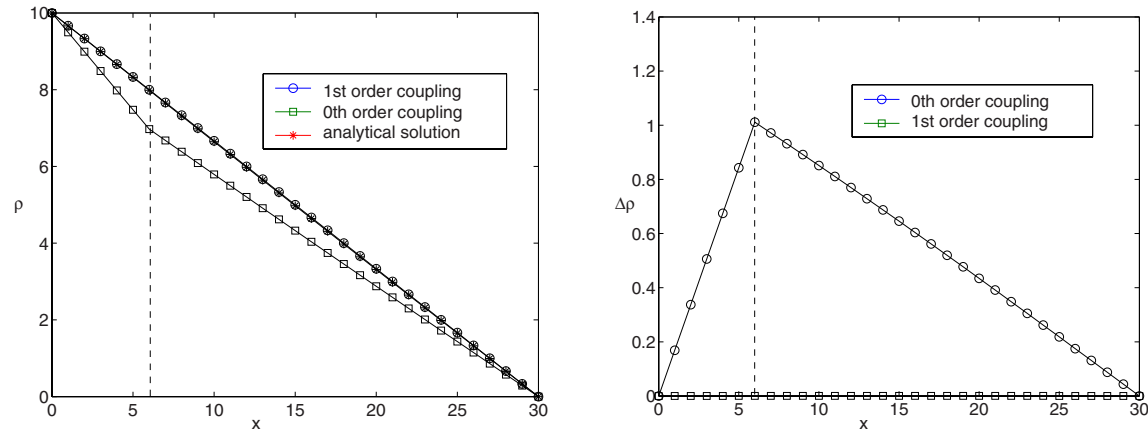

Fig. 2. The density profile along the horizontal axis of the domain $\Omega$ is plotted on the left side and the error with respect to the analytic solution on the right side. The squares correspond to a simulation in which the coupling algorithm does not include the gradient correction, whereas the circles represent the case where the full coupling is considered. The asterisks of the analytic solution are diffcult to distinguish from the circles of the full coupling. The total grid size is $31 \times 31$, and there are respectively (a)100, (b)500 and (c)5000 time steps. The vertical interface (dashed line) is located at $x=6$. The relaxation time is $\tau=0.7$, the lattice spacing $\Delta r=1$ and the time step $\Delta t=10^{-1}$ 
with a FD solver on the other. At the interface, the LB and FD are connected so as to preserve continuity of the physical quantities. The connection between the $f_{i}$ s and the standard macroscopic physical quantities is obtained using a multiscale Chapman-Enskog expansion to split the $f_{i}$ s in an equilibrium and a nonequilibrium part. The former is related to the particle density and the latter to its gradient. Our coupling methodology is indeed an approximation since we neglect higher-order derivatives in the nonequilibrium distributions. A validation was performed by running numerical simulations on a 2D domain and comparing them with an analytic solution. Our mapping has the same level of accuracy as the LB itself: second order in the lattice spacing.

Regarding future work, it seems natural to try to couple two domains with different meshes. A good enough interpolation is needed to keep the second-order accuracy of the coupling scheme (see [2]). This as well as the case of an irregular boundary is under investigation. Other perspectives include applying the same methodology to the LB reaction-diffusion, wave and fluid models. We also wish to couple different LB dynamics: a diffusive region with a convective one. With respect to coupling an incompressible Navier-Stokes solver with a "compressible" LB fluid model, we believe that the pressure obtained from the Navier-Stokes solver can be used to specify the density of the LB scheme, and conversely. We plan to examine this on a Poiseuille flow and on Blasius' problem.

\section{References}

1. B. Chopard, P.O. Luthi, A. Masselot, and A. Dupuis. Cellular automata and lattice Boltzmann techniques: An approach to model and simulate complex systems. Advances in Complex Systems, 5(2), 2002. http://cui.unige.ch/ chopard/FTP/CA/advp.ps.gz.

2. A. Dupuis and B. Chopard. Theory and applications of alternative lattice Boltzmann refinement algorithm. Phys. Rev. E, 67:066707, 2003. In press.

3. L. Beilina. A hybrid method for elastic waves. Technical report, Finite Element Center, Chalmers University of Technology, 2003. Preprint 2003-01.

4. A. Malevanets and R. Kapral. Mesoscopic model for solvent dynamics. J. of Chem. Phys., 110(17):8605-8613, May 1999.

5. D.A. Wolf-Gladrow. Lattice-Gas Cellular Automata and Lattice Boltzmann Models: an Introduction. LNM, 1725. Springer, Berlin, 2000.

6. B. Chopard and M. Droz. Cellular automata model for diffusion processes. J. Stat. Phys., 64:859-892, 1991.

7. R. G. M. Van der Sman and M. H. Ernst. Convection-diffusion lattice boltzmann scheme for irregular lattices. J. Comp. Phys., 160:766-782, 2000. 